\title{
Functional differentiation related to decomposing complex carbohydrates of intestinal microbes between two wild zokor species based on 16SrRNA sequences
}

Yao Zou, Nannan Liang, Xuxin Zhang, Chongxuan $\operatorname{Han}^{*}$ (I) and Xiaoning Nan ${ }^{*}$

\begin{abstract}
Background: The intestinal microbes in mammals play a key role in host metabolism and adaptation. As a subterranean rodent, zokor digs tunnels for foraging and mating. These digging activities of zokors increase the energy expenditure relative to their aboveground counterparts. However, relatively little is known regarding intestinal microbes of zokor and how they make full use of limited food resources underground for high energy requirements.
\end{abstract}

Results: Eospalax cansus and Eospalax rothschildi had distinct intestinal microbes. Although the composition of intestinal microbes is similar in two species, the proportion of bacterium are distinctly different between them. At phylum level, 11 phyla were shared between two species. Firmicutes and Bacteroidota were two dominant microbes in both of two species, while Eospalax cansus have a significantly high proportion of Firmicutes/ Bacteroidota than that of Eospalax rothschildi. At genus level, norank_f_Muribaculaceae were dominant microbes in both of two zokor species. The relative abundance of 12 genera were significantly different between two species. Some bacterium including unclassified_f_Lachnospiraceae, Lachnospiraceae_NK4A136_group, Ruminococcus and Eubacterium_siraeum_group associated with cellulose degradation were significantly enriched in Eospalax cansus. Although alpha diversity was with no significant differences between Eospalax cansus and Eospalax rothschildi, the intestinal microbes between them are significant distinct in PCoA analysis. We have found that trapping location affected the alpha diversity values, while sex and body measurements had no effect on alpha diversity values. PICRUSt metagenome predictions revealed significant enrichment of microbial genes involved in carbohydrate metabolism in Eospalax cansus rather than Eospalax rothschildi.

Conclusions: Our results demonstrate that Eospalax cansus harbor a stronger ability of fermentation for dietary plants than Eospalax rothschildi. The stronger ability of fermentation and degradation of cellulose of intestinal microbes of Eospalax cansus may be a long-time adaptation to limited food resources underground.

Keywords: Zokor, Intestinal microbes, Fermentation, Cellulose, Adaptation

\footnotetext{
*Correspondence: 1139186993@qq.com; 358727493@qq.com

Key Laboratory of National Forestry and Grassland Administration on Management of Western Forest Bio-Disaster, Northwest Agriculture and Forestry University, Yangling 712100, China
}

(c) The Author(s). 2021 Open Access This article is licensed under a Creative Commons Attribution 4.0 International License, which permits use, sharing, adaptation, distribution and reproduction in any medium or format, as long as you give appropriate credit to the original author(s) and the source, provide a link to the Creative Commons licence, and indicate if changes were made. The images or other third party material in this article are included in the article's Creative Commons licence, unless indicated otherwise in a credit line to the material. If material is not included in the article's Creative Commons licence and your intended use is not permitted by statutory regulation or exceeds the permitted use, you will need to obtain permission directly from the copyright holder. To view a copy of this licence, visit http://creativecommons.org/licenses/by/4.0/ The Creative Commons Public Domain Dedication waiver (http://creativecommons.org/publicdomain/zero/1.0/) applies to the data made available in this article, unless otherwise stated in a credit line to the data. 


\section{Background}

Zokor is one of subterranean rodents endemic to east Asia [1]. It gnaw the roots of plants for food and could cause large-scale disasters to woodland and meadow [2]. Due to its special underground lifestyle, it is difficult to capture zokor so that the study of zokor is limited. More attentions are paid to the classification and phylogeography of zokor [3-6], while the researches on physiology and ecology of zokor are scarce. As a subterranean rodent, zokor has harbored many adaptive characteristics during long-time process of evolution, such as vestigial eyes, strong forelimbs $[7,8]$ and developed cecum [9, 10]. However, there are interspecies differences in these adaptive characteristic due to the differences of genetic background and living conditions. It is of great significance to compare and study the adaptive characteristics of different species of zokor.

The genomes of intestinal microbes in mammals contain more than 100 times as many genes as the host genomes [11]. The diversity of intestinal microbes plays an important role in maintaining the intestinal microflora and body balance. The composition and metabolites of intestinal microbes are closely related to the health status of host. Intestinal microbes have many physiological functions, they not only affect the digestion and absorption of food, but also regulate nutrition and immune response of host, and help the host to obtain energy from food [12]. One of the core functions is to help the host to decompose the complex polysaccharides which escaped from digestion of foregut [13]. As a phytophagous mammal, developed cecum of zokor indicates the morphological adaptation of digestive organs [10]. It is suggested that zokor has strong fermentation ability for complex polysaccharides because the expansion of cecum can extend the residence time of food in gut and thus improve the utilization of food [9]. However, there are little researches on intestinal microbes of zokor, and how limited food resources underground are fully utilized by intestinal microbes of zokor is still unknown. It is worth investigating the intestinal microbes of zokor and comparing the differences between different zokor species.

Intestinal microbes and hosts together formed relatively stable mutualism in the long-time process of evolution. Firmicutes, Bacteroidota and Actinobacteria are three dominant microbes of mammals [14]. Intestinal microbes of mammal are mainly influenced by host and environment factors $[14,15]$. Host factors include genetic background, age, gender and the health status of host, while environment factors include food, season, environment microbes and geographical location. It was found that the season had significant impact on both community structure and diversity of cecal bacteria of Eospalax cansus (E. cansus). And this seasonal variation of zokor intestinal microbes was related to seasonal changes in food resource [16]. By feeding E. cansus on different types of plants, it was found that different food composition influence gut microbiota of zokor [17]. Zokors fed by woody plants have a higher proportion of Firmicutes/Bacteroidota and higher diversity compared to zokors fed by herbs. High proportion of Firmicutes/ Bacteroidota of intestinal microbes of zokor was a response to the high-fiber food resources, and it would help zokor to obtain more energy from dietary plant.

Extant zokors (Myospalacinae) consist of two genera, Eospalax and Myospalax [4, 18]. Zokors in Eospalax are endemic species of China. Molecular evidences based on mitochondrial genome suggested that E. cansus and Eospalax rothschildi ( $E$. rothschildi) are closely related in Eospalax [19]. However, the living conditions of habitats between these two species are distinct. E. cansus lives in the Loess Plateau area $\left(34.68-41.60{ }^{\circ} \mathrm{N}, 100.55-107.94\right.$ $\left.{ }^{\circ} \mathrm{E}\right)$ located in the northwest of China where the vegetation is sparse [20], and it often takes roots of woody plants as food because edible plant resources are limited. Differently, E. rothschildi lives in Qinba mountain area $\left(31.13-32.60{ }^{\circ} \mathrm{N}, 107.55-113.06{ }^{\circ} \mathrm{E}\right)$ located in the southcentral region of China, where the habitat is with relatively high annual mean temperature, plentiful rainfall, mixed evergreen and deciduous broad-leaved forest [21]. The edible types of plants for $E$. rothschildi are relatively abundant and various, including woody plants and herbaceous plants. The composition and structure of intestinal microbes of these two species and influencing factors seem to be unclear. This study investigated the composition and diversity of intestinal microbes of $E$. cansus and E. rothschildi, and compared the differences of intestinal microbes between these two species. Furthermore, the functions of intestinal microbes of $E$. cansus and E. rothschildi were predicted to reveal the functional differentiation of intestinal microbes between two zokor species. These findings would provide novel insights into the contributions of intestinal microbes to adaptive evolution of zokors and the process of species differentiation of Myospalacinae.

\section{Results}

\section{Composition and relative abundance of intestinal} microbes between $\mathrm{E}$. cansus and $E$. rothschildi

After filtering out low-quality sequences, chloroplasts, chimeras and singletons, we obtained $2,138,122$ valid sequences from 55 samples. To standardize sampling efforts across samples, each sample was rarefied to 22,494 sequences. With the increase of data size, the OTU-level rarefaction curves (Fig. S1) gradually reached stable values, indicating that the sequencing depth has reached the requirement. A total of 1796 OTUs, 12 phyla and 180 genera were detected in the gut microbiota of all 
zokors, and 1233 OTUs, 11 phyla and 133 genera were shared in two species, respectively (Fig. S2).

At phylum level, the zokor gut bacteriome across all samples were dominated by Firmicutes and Bacteroidota, followed by Desulfobacterota, Actinobacteriota and Proteobacteria (>1\% relative abundance) (Fig. 1). We compared the difference of gut microbiota composition between $E$. cansus and E. rothschildi. E. cansus has more microbes than that of E. rothschildi at OTU, phylum or genus level (Fig. S2). Firmicutes and Bacteroidota were dominant microbes in both of two species (Fig. 1). However, there are some differences in proportion between two species at phylum level. The proportion of Firmicutes was 69.97 and 51.31\%, while Bacteroidota was 22.77 and $35.90 \%$ in E. cansus and E. rothschildi, respectively (Fig. 1). Firmicutes were more abundant in $E$. cansus, while the relative abundance of Bacteroidota, Desulfobacterota and Actinobacteriota were significantly higher in E. rothschildi. (Fig. 3). In addition, E. cansus harbored one unique Verrucomicrobia $(<1 \%$ relative abundance). The bacteria of this phylum were identified as genus Akkermansia.

At genus level, norank_f_Muribaculaceae were dominant microbes in both of two species, and unclassified_f_ Lachnospiraceae, Lachnospiraceae_NK4A136_group, norank_f_Oscillospiraceae and Desulfovibrio were also enriched in two zokor species (Fig. 2). The proportion of norank $f \_$Muribaculaceae was 21.59 and $35.20 \%$ in E. cansus and E. rothschildi, respectively (Fig. 2). There were 12 genera significantly different between two species (Fig. 4). unclassified_f_Lachnospiraceae, Lachnospiraceae_NK4A136_group, Ruminococcus, Eubacterium siraeum_group and Lactobacillus were enriched in $E$. cansus, while norank $f \_$Muribaculaceae, norank $f$ Christensenellaceae, unclassified_f_Christensenellaceae, Oscillospiraceae, Desulfovibrio, norank $f \_$Oscillospiraceae and NK4A214_group were more abundant in $E$. rothschildi. In addition, E. cansus harbored 36 unique genera, and the bacteria with relative abundance $>0.1 \%$ were Paraclostridium, Vagococcus, Actinobacillus and unclassified_f_Prevotellaceae. E. rothschildi harbored 11 unique genera, and the bacteria with relative abundance $>0.1 \%$ were Treponema and unclassified_c_Bacteroidia.

\section{Alpha diversity}

Alpha diversity was estimated by four indices including Shannon, Chao, ACE and Simpson. We have found that there were no significant differences $(p>0.05)$ between two species on these four indices by Wilcoxon rank-sum test (Fig. S3).

We investigated whether trapping location, sex, and body measurements affected the alpha diversity values. There was a significant effect of trapping location on Chao and ACE indices within E. cansus $(p<0.05$; Table 1). Samples from PY had significantly greater Chao $(p<0.01)$ and ACE $(p<0.05)$ indices than that of LD. However, there was no significant effect of trapping location on the Shannon and Simpson diversity. Besides,

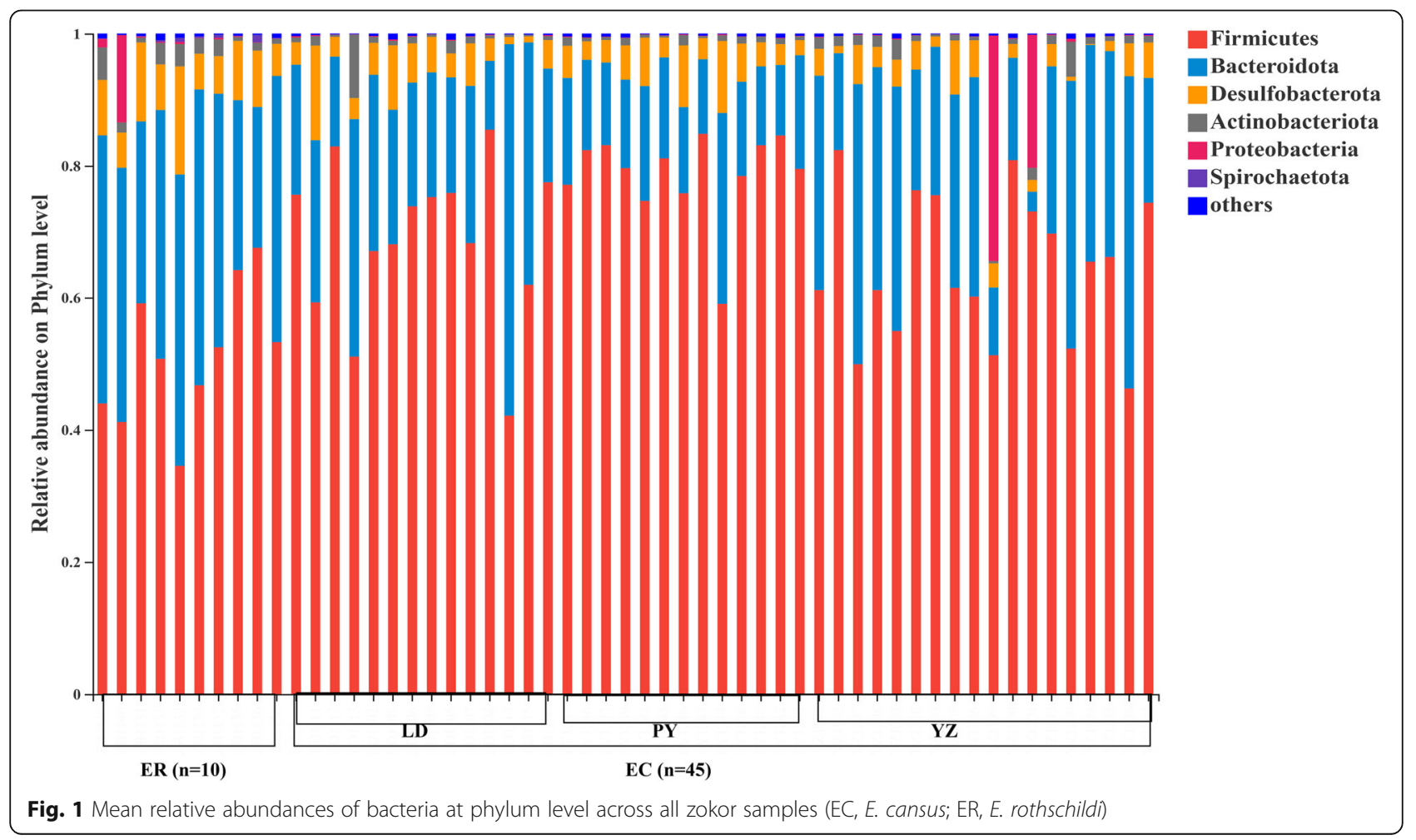




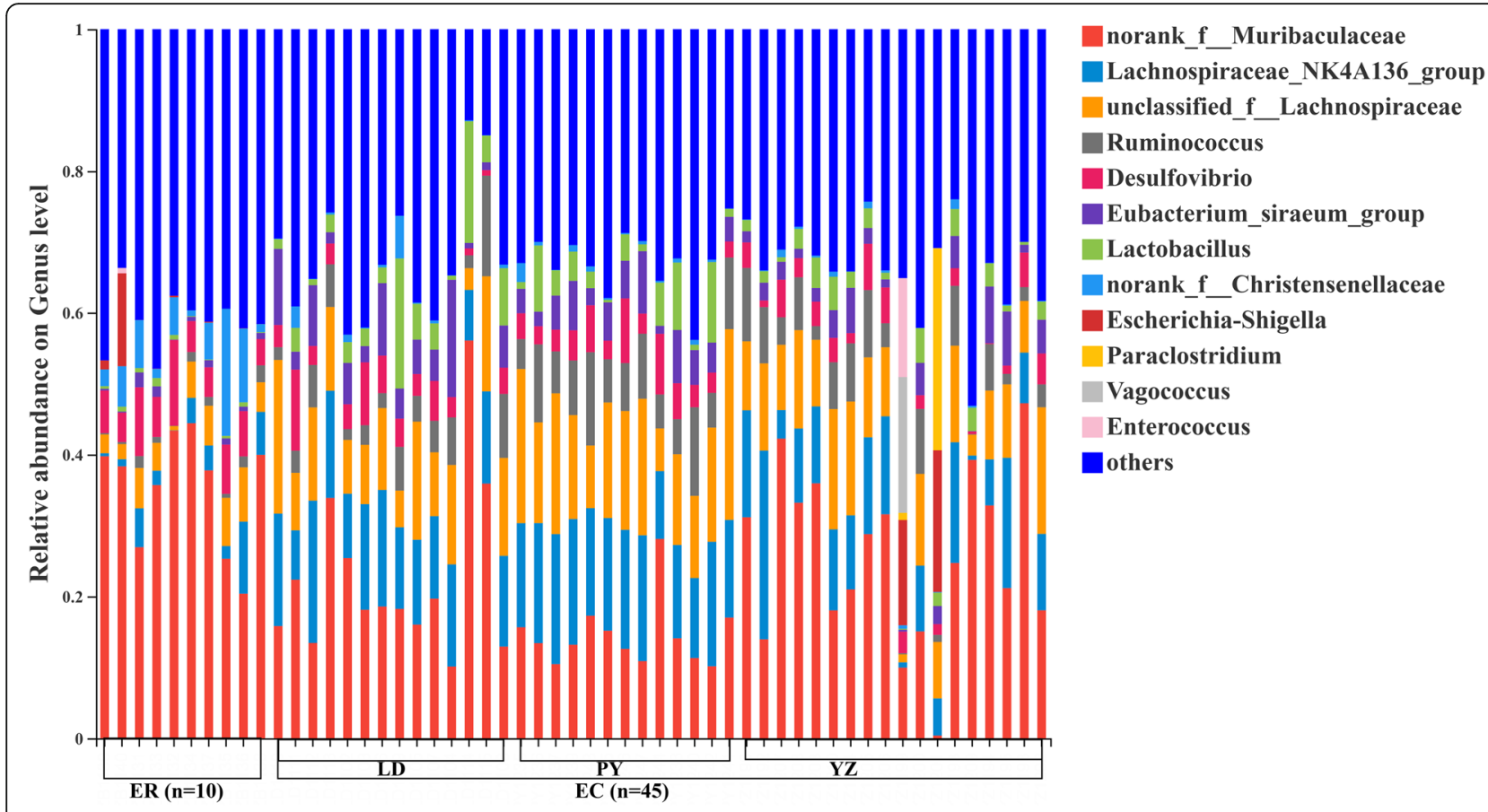

Fig. 2 Mean relative abundances of bacteria at genus level across all zokor samples (EC, E. cansus; ER, E. rothschildi)

sex had no significant on alpha diversity of E. cansus $(p>0.05$; Fig. S4). Sex had no significant on Shannon, Chao and ACE indices of E. rothschildi ( $p>0.05$; Fig. S5), however, males have greater Simpson index than that of females $(p<0.05)$.

The body measurements including weight, body length, weight/body length and tail length for each sample were shown in Table S1. We also searched for correlations (Pearson with two-tailed significance tests) between the alpha diversity and body measurements of E. cansus and E. rothschildi, respectively. We didn't find the significant correlations between the values of alpha diversity and body measurements including weight, body length, weight/body length and tail length in both of two zokor species $(p>0.05$; Table S2).

\section{Beta diversity}

To intuitively demonstrate the differences of intestinal microbes between two species, principal coordinate analysis (PCoA) was performed based on Bray-Curtis and unweighted UniFrac distance to visualize the separation of gut microbiota structure across different species. By ranking the distance among samples, the order of samples in the low dimensional space can reflect the relationship among them. Principal coordinate analysis showed that the E cansus and E. rothschildi had distinct composition of intestinal microbes (Fig. 5). ANOSIM analysis confirmed that the structure of gut microbiota was significantly influenced by host species (Bray-Curtis, $r=0.649, p<0.001$; unweighted UniFrac, $r=0.742, p<$ 0.001 ), while sex had no significant effect on the gut microbiota $(p>0.05)$.

\section{Predicted metagenomes}

The function of microbes of zokor based on COGs include carbohydrate transport and metabolism, transcription, amino acid transport and metabolism and other 21 functions (Table S3). The relative abundances of most of functions gene categories were extremely significantly higher in $E$. cansus than those in E. rothschildi $(p<0.01)$

Table 1 Mean alpha diversity ( \pm SD) values for each trapping location. Letters after the values denote groups that differ significantly at $p<0.05$ for alpha diversity

\begin{tabular}{lllll}
\hline Trap site & Shannon & Chao & ACE & Simpson \\
\hline LD & $5.00(0.48)$ & $895.74(67.61) \mathrm{a}$ & $880.19(67.08) \mathrm{a}$ & $0.023514(0.0217)$ \\
PY & $5.30(0.13)$ & $971.43(70.27) \mathrm{b}$ & $948.58(65.66) \mathrm{b}$ & $0.014551(0.0037)$ \\
YZ & $4.96(0.70)$ & $925.99(142.03) \mathrm{ab}$ & $908.44(132.83) \mathrm{ab}$ & $0.026558(0.0339)$ \\
\hline
\end{tabular}


except for extracellular structures $(p>0.05)$. Thereinto, carbohydrate transport and metabolism was the most important function.

\section{Discussion}

\section{Intestinal microbes of zokor accord with the} characteristics of herbivores

In this study, the composition and structure of intestinal microbes of $E$. cansus and $E$. rothschildi were studied by high-throughput sequencing technology. At phylum level, Firmicutes and Bacteroidota are dominant microbes in both of two zokor species. The total proportion of Firmicutes and Bacteroidota are 92.74 and $87.21 \%$ in E. cansus and E. rothschildi, respectively (Fig. 3). Firmicutes and Bacteroidota are mainly responsible for food fermentation in the gut [22]. High percentage of Firmicutes and Bacteroidota contributes to better decomposition of the cellulose and hemicellulose, and it accords with the characteristics of herbivores such as horse and donkey [23, 24]. At genus level, norank $f \_$Muribaculaceae are dominant genera in both of two species (Fig. 4). Norank $f_{-}$Muribaculaceae belong to the family S24-7, and S24-7 spp. are related to the degradation of a variety of complex carbohydrates [25]. Therefore, the dominant composition of intestinal microbes of $E$. cansus and $E$. rothschildi at phylum and genus level indicates that intestinal microbes of zokor are highly adaptive to phytophagous habits.

\section{Diversity of intestinal microbes and its correlation of two} zokor species

Intestinal microbial diversity is an important indicator of stability and metabolic capacity [26, 27]. Intestinal microbes with high diversity provide a stronger capacity to utilize various metabolic pathways [28]. Although the composition of microbes of E. cansus were more abundant than that of $E$. rothschildi at OTU, phylum or genus level (Fig. S2), but it's related to the larger sample size of E. cansus. Shannon, Chao, ACE and Shannon indices were with no significant differences between $E$. cansus and E. rothschildi (Fig. S3). It is suggested that the intestinal microbes of both two zokor species might be stable and have many functions. Beta diversity result (Fig. 5) showed that the samples of $E$ cansus and the samples of $E$. rothschildi were clearly separated, demonstrated the structural divergence of intestinal microbes between two zokor species. Moreover, we have found that the composition and structure of intestinal microbes of $E$. cansus were more similar to each other than that of $E$. rothschildi regardless of geographical location, showing that the interspecific distance was larger than intraspecific distance. It is also suggested that the genetic background of host is an important factor in shaping intestinal microbes $[29,57]$. Although two zokor species are closely related, the habitat types and diet resources were distinct between two zokor species. All of these factors may contribute to the divergence of intestinal

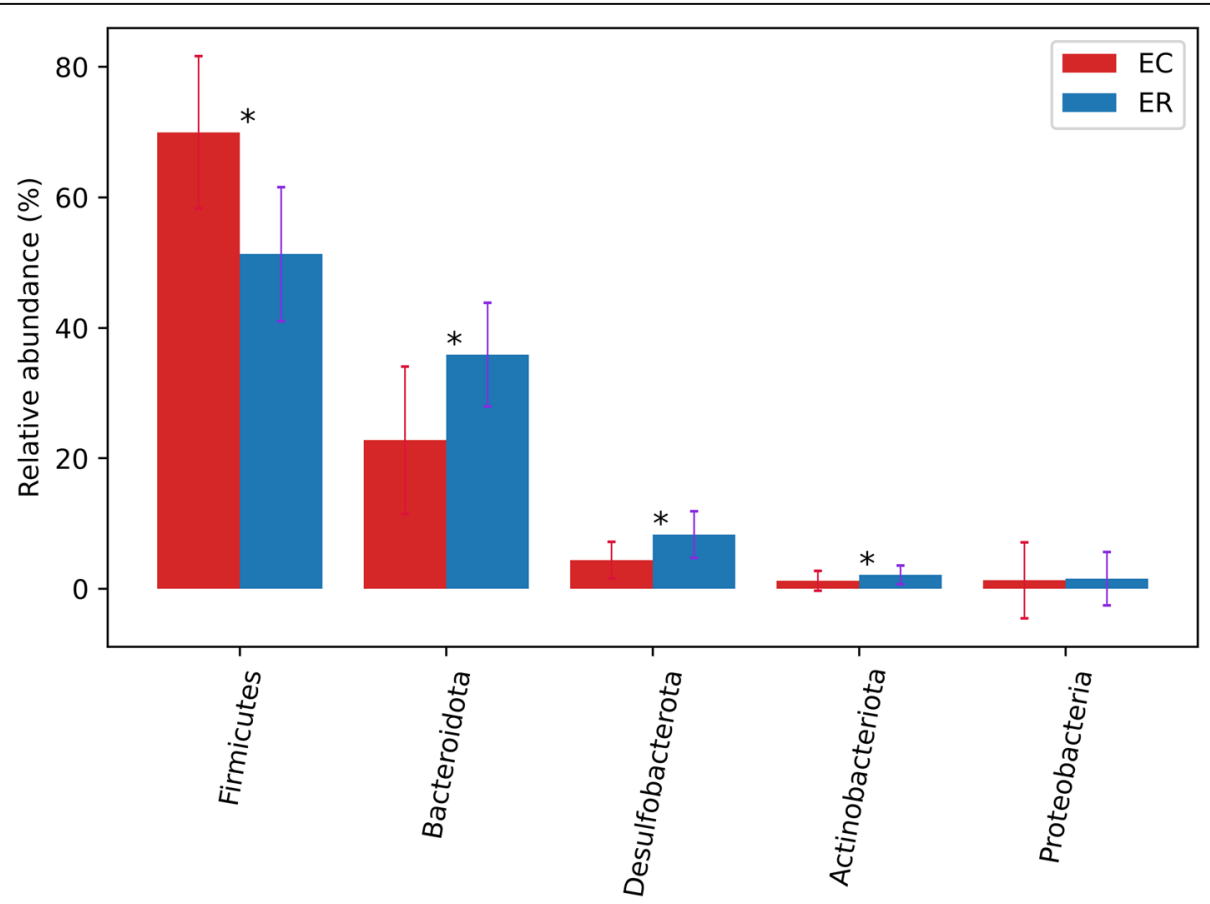

Fig. 3 Differences of composition of intestinal microbes between two zokor species at phylum level (EC, E. cansus; ER, E. rothschildi). Significant difference is indicated by asterisk. ${ }^{*}, p<0.05 ;{ }^{* *}, p<0.01$; ${ }^{* *}, p<0.001$ 


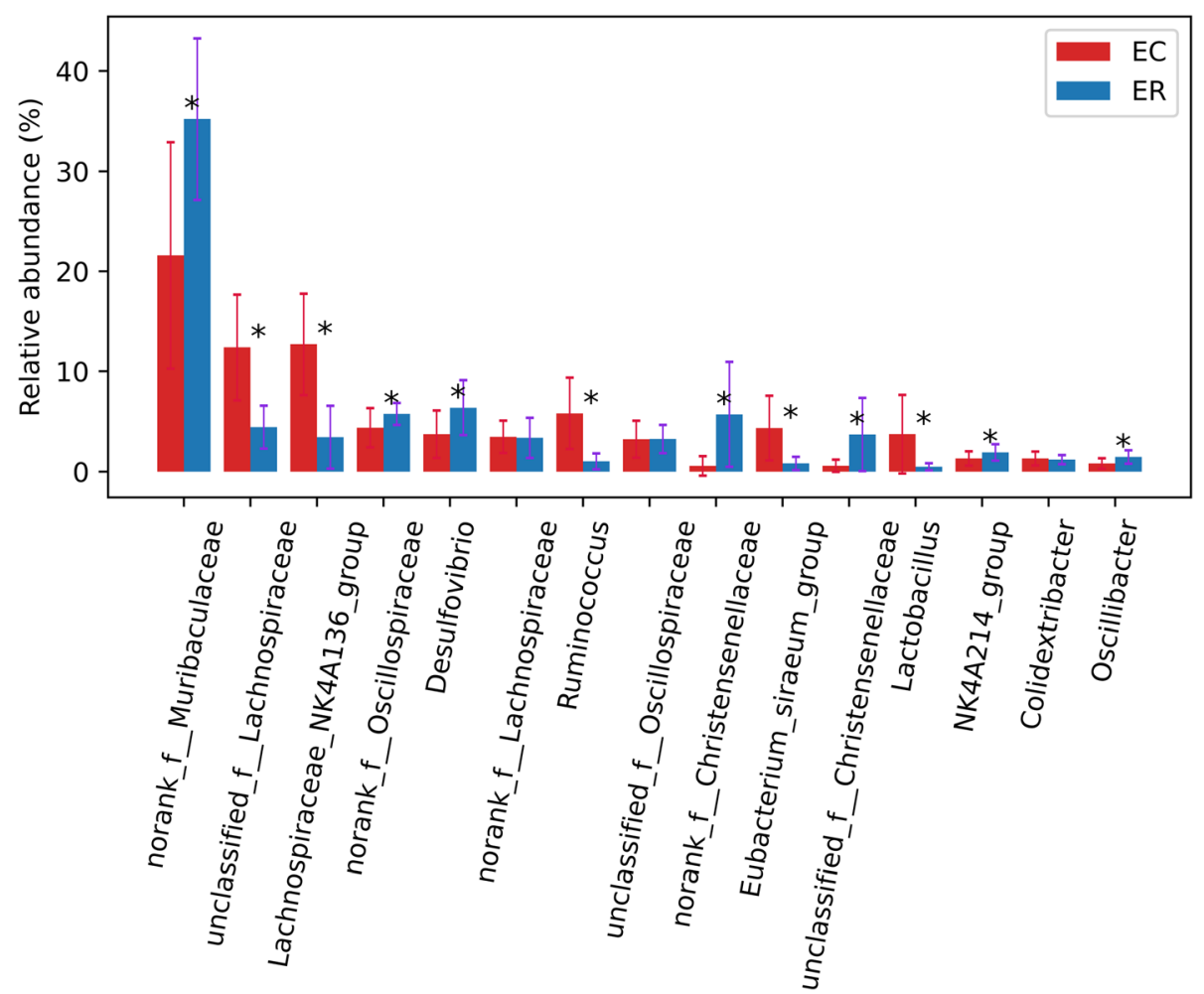

Fig. 4 Differences of composition of intestinal microbes between two zokor species at genus level (EC, E. cansus; ER, E. rothschildi). Significant difference is indicated by asterisk. ${ }^{*}, p<0.05 ;{ }^{* *}, p<0.01$; ${ }^{* *}, p<0.001$

bacteriome. Correspondingly, similar results have been found in pika [29] and ruminants [30].

To explore the influencing factor to alpha diversity, we found that there was a significant effect of trapping location on the alpha diversity (Table 1). E. cansus in LD had lower bacterial diversity than that of PY, which may be due to lower diet diversity of $E$. cansus resulted by lower diversity of plant communities in LD. In fact, we just observed significant differences on Chao and Ace indices. Chao and Ace indices $[65,66]$ could reflect the richness of bacterial communities while Shannon and Simpson $[63,64,67]$ could reflect the diversity of bacterial communities. It is inferred that the diversity of bacterial species may not be easier to change than richness despite the far geographical distance between these two populations. We didn't find significant effect of sex on alpha diversity of two zokor species (Fig. S4; Fig. S5). These results were consistent with precious study [16]. However, we only observed the differences of Simpson indices between two sexes in E. rothschildi, which may be resulted from small sample size of E. rothschildi. In addition. This was the first research on exploring the correlations between the alpha diversity and body measurements of zokor. We didn't find the significant correlations between them (Table S2). However, significant correlations of microbiota diversity and body measurements including weight, body-mass index (weight/body length) were found in Mus musculus [31]. To make our results more reliable, more samples of zokors especially E. rothschildi should be collected and supplemented in further study.

\section{Comparison of intestinal microbes composition between two zokor species}

Although the composition of intestinal microbes is similar in E. cansus and E. rothschildi, the proportion of bacterium are distinctly different between two species. The relative proportion of Firmicutes and Bacteroidota varied between the two zokor species (Fig. 3). E. cansus have a high proportion of Firmicutes/Bacteroidota than that of E. rothschildi. It is reported that high proportion of Firmicutes/Bacteroidota was related to carbohydrates diet [32], and was also related to obesity [33]. Firmicutes/ Bacteroidota is usually used as an indicator to measure the status of obesity [34]. In the sampling process, we also found that the body size and fat thickness of $E$. rothschildi was significantly lesser than that of E. cansus. It is suggested that high proportion of Firmicutes/Bacteroidota of $E$. cansus contributes to obtaining more energy from dietary plants, especially complex carbohydrates. Verrucomicrobia were unique phylum in E. cansus. Species of Akkermansia (phylum 

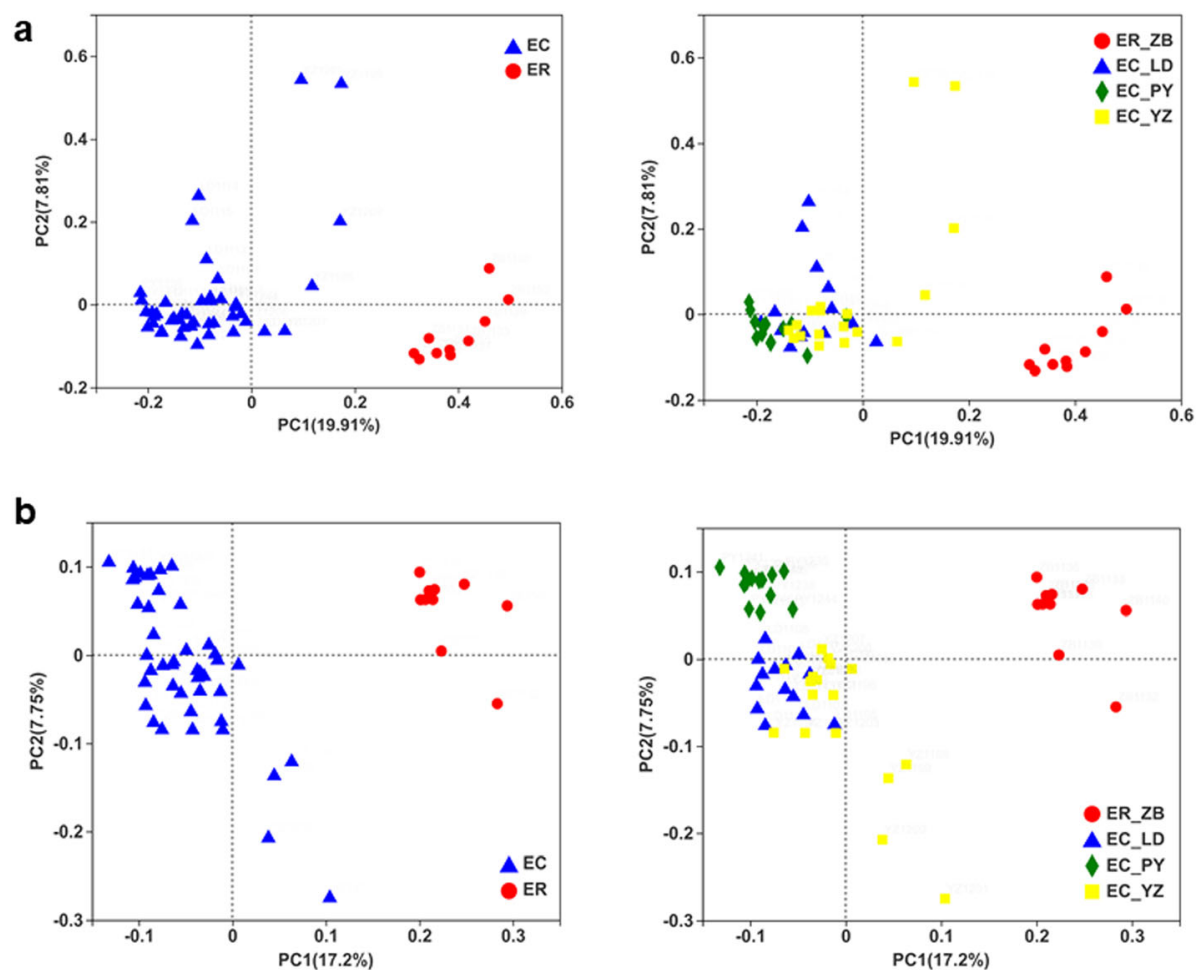

Fig. 5 Principal coordinates analysis (PCOA) of bacterial communities of two zokor species across 55 samples. a. based on Bray-Curtis distance metrics $(r=0.649, p<0.001)$. b. based on the unweighted UniFrac distance metrics $(r=0.742, p<0.001)$. Note: $E C$, E. cansus; ER, E. rothschildi; ER_ZB: E. rothschildi in ZB; EC_LD: E. cansus in LD; EC_PY: E. cansus in PY; EC_YZ: E. cansus in YZ

Verrucomicrobia) are mucin-degrading bacteria, which may be capable of using mucin as carbon source for host [35]. Zokor especially E. cansus often faced with the lack of food resources due to its underground lifestyle, the Akkermansia within E. cansus may be beneficial for zokor to obtain energy from mucin.

At genus level (Fig. 4), the genera with significantly high proportion in E. cansus were related to the degradation of complex carbohydrates such as cellulose and hemicellulose. Unclassified_f_Lachnospiraceae and Lachnospiraceae_NK4A136_group both belong to Lachnospiraceae which are involved in metabolism as butyrate producer [36, 37]. And the latter was fibrolytic bacterium which can degrade the complex plant bran of recalcitrant substrate [38,39]. Besides, species in Ruminococcus like Ruminococcus albus and Ruminococcus flavefaciens were widely studied in herbivores, and these two species have played a key role in decomposition of cellulose and hemicellulose [40-42]. In addition, Eubacterium_siraeum_group were proven to have the ability to ferment cellobiose to acetic acid [43], and Lactobacillus which have probiotic functions can ferment the carbohydrates to lactic acid [44]. It is indicated that intestinal microbes of $E$. cansus have stronger ability to ferment complex carbohydrates than that of E. rothschildi. Compared with $E$. rothschildi rich in edible plants, E. cansus just used limited resources as diet such as the roots of woody plants. These woody plants generally may have higher crude fiber than herbaceous plants [17]. Therefore, stronger ability of fermentation and degradation of cellulose of $E$. cansus is a long-time adaptation to limited food resources of its habitat. We have found significantly decreased NK4A214_group in $E$. cansus compared to $E$. rothschildi. Similar results have also been found in pigs, it is shown that the relative abundance of NK4A214_group were decreased when pigs were fed by wheat bran (fibrous) [45].

For $E$. rothschildi, the genera which were enriched in this species were related to the catabolism of various ingredient. Norank $f_{-}$Muribaculaceae might be related to the degradation of a variety of carbohydrates [25]. Norank_f_Christensenellaceae and unclassified_f_Christensenellaceae belong to the family Christensenellaceae, and species of this family has many physiological functions such as catabolism of protein and prebiotic fibers [4649]. In addition, Oscillospiraceae and norank_f_Oscillospiraceae could utilize effectively carbohydrates and monosaccharide of tender leaves and roots [50]. Furthermore, species of Desulfovibrio were a group of sulphatereducing bacterium which can decompose sulfate into hydrogen sulphide [51]. These bacteria enriched in E. rothschildi indicated the adaptation to the abundant food resources of its habitat. 
Unique genera in E. cansus including Paraclostridium, Vagococcus, Actinobacillus and unclassified_f_Prevotellaceae were related to emerging pathogens [52-55]. Unique genus Treponema in E. rothschildi were also related to pathogens [56], however, the functions of unclassified_c_Bacteroidia were unknow. These unique genera of two zokor species may be host-specific bacteria, which may result from species loss and sorting due to the enrichment of nutrients [57].

PICRUSt metagenomic prediction showed that the relative abundances of the carbohydrate metabolism gene categories were significantly higher in $E$. cansus than those in E. rothschildi (Table S3). It is inferred that intestinal microbes of $E$. cansus would help hosts to maximize the nutrient utilization and energy extraction from indigestible plant carbohydrates, such as cellulose and hemicellulose.

This is the first time to report the composition and structure of intestinal microbes of $E$. rothschildi, however, the intestinal microbes of E. cansus was first reported in 2018 [16]. In this study, the intestinal microbes of $E$. cansus and $E$. rothschildi were investigated. It is shown that the abundance and diversity of intestinal microbes are distinct between two species, although there are little differences in composition of intestinal microbes. Furthermore, the intestinal microbes of these two species gradually differentiated in some functions especially the degradation of complex carbohydrates. These works would be significant to understanding the adaptive evolution of zokor and the process of species differentiation of Myospalacinae.

\section{Conclusions}

In conclusion, our study demonstrates that the intestinal microbes between Eospalax cansus and Eospalax rothschildi. Are distinct, although there are little differences in composition of intestinal microbes. Eospalax cansus harbor a stronger ability of fermentation for dietary plants than that of Eospalax rothschildi. The stronger ability of fermentation and degradation of cellulose of intestinal microbes of Eospalax cansus may be a long-time adaptation to limited food resources underground.

\section{Methods}

\section{Sample collection}

Samples were collected from May to July in 2020. Wild adult E. cansus and E. rothschildi were captured from Ningxia Hui Autonomous Region and Shaanxi Province of China, respectively. The details of sampling information were shown in Table 2. After zokors were humanely euthanized by intravenous pentobarbital sodium (390 $\mathrm{mg} / \mathrm{mL}$ ) overdose after sedation with xylazine hydrochloride $(5 \mathrm{mg} / \mathrm{kg})$. The cecal contents were instantly collected from the ceca of wild zokors within $5 \mathrm{~min}$ of euthanasia, and immediately placed in cryogenic vials, frozen in liquid nitrogen, and then stored at $-80^{\circ} \mathrm{C}$ in a refrigerator in the laboratory. 55 cecal samples were obtained from two species of zokors, including $E$. cansus $(n=45)$ and $E$. rothschildi $(n=10)$. All animal experiments were approved by the Institution of Animal Care. Processing of wild animals and sample collection were strictly congruent with the guidelines of our academic institution.

The major plant community in each sampling site was identified based on morphological characteristics. Herbaceous plants including different families were dominated by Rubus corchorifolius, Artemisia argyi, Plantago depressa, Viola diffusa and Rehmannia glutinosa in the habitat of E. rothschildi in Zhenba county (ZB). However, there were less plant species in the habitat of $E$. cansus, and woody plants were major composition. Economic forests are being developed over the land of $E$. cansus habitat in Longde county (LD). As a result, there were fewer weeds and lower diversity of plant communities. The plant community in LD were dominated by Corylus heterophylla, Hemerocallis citrina and Isatis tinctorial. Malus pumila and Isatis tinctorial were dominant plants in Pengyang county (PY), while Larix gmelinii, Amygdalus davidiana, Hippophae rhamnoides and Urtica fissa were dominant plants in Yuanzhou distinct (YZ). The phloem of roots even the entire root of these arbors and shrubs, and the grass roots and rhizomes of these herbaceous plants were used as the diets of zokors.

\section{DNA extraction}

Total genomic DNA of zokor was extracted with a Stool Genome DNA Extraction Kit (Tiangen Inc.) from the

Table 2 Information of sampling area of zokor

\begin{tabular}{|c|c|c|c|c|c|c|}
\hline Sampling locality & Species & Code & Longitude $/{ }^{\circ} \mathrm{E}$ & $\begin{array}{l}\text { Latitude } \\
\text { / }^{\circ} \mathrm{N}\end{array}$ & Altitude/m & $\begin{array}{l}\text { Sample size } \\
\text { (male/female) }\end{array}$ \\
\hline Shenlin forest in Longde county & E. cansus (EC) & LD & 105.9290 & 35.579 & 1824 & $14(8 / 6)$ \\
\hline Xiaoshigou village in Pengyang county & E. cansus (EC) & PY & 106.8456 & 36.0273 & 1721 & $13(3 / 10)$ \\
\hline Hongzhuang forest in Yuanzhou & E. cansus (EC) & YZ & 106.1143 & 35.8121 & 2202 & $18(12 / 6)$ \\
\hline Xingzi forest in Zhenba county & E. rothschildi (ER) & ZB & 108.0207 & 32.5008 & 1624 & $10(4 / 6)$ \\
\hline
\end{tabular}


cecal contents following the manufacturer's protocol. DNA concentration and quality were determined using the Nanodrop 2000 Spectrophotometer (Thermo Scientific, Wilmington, USA). DNA were detected with $1 \%$ agarose gel extraction kit (Takara Inc.) and then purified and sequenced by Majorbio Bio-Pharm Technology Co. Ltd. (Shanghai, China).

\section{PCR amplification and MiSeq sequencing of 16S rRNA gene}

The universal primer pair (338 F: 5'-ACTCCTACGG GAGGCAGCAG-3', 806 R: 5'-GGACTACHVGGG TWTCTAAT $-3^{\prime}$ ) was used to amplify the $16 \mathrm{~S}$ rRNA (V3-V4 hypervariable regions) by PCR system (GeneAmp 9700, ABI, USA) from cecal contents DNA [58]. The procedures of PCR amplification, with $25 \mu \mathrm{L}$ reaction volume, including $1 \mu \mathrm{L}$ DNA, $0.5 \mu \mathrm{L}$ dNTPs mix (10 mM concentration of each dNTP), $5 \times$ High GC Buffer $5 \mu \mathrm{L}, 1.0 \mu \mathrm{L}$ of each primer $\left(10 \mu \mathrm{mol} \cdot \mathrm{L}^{-1}\right)$ and $0.25 \mu \mathrm{L}$ Q5 high-fidelity DNA polymerase, with sterile distilled water added up to $25 \mu \mathrm{L}$ volume. Amplification conditions were $3 \mathrm{~min}$ at $95^{\circ} \mathrm{C}$ followed by 27 cycles of $95^{\circ} \mathrm{C}$ for $30 \mathrm{~s}, 55^{\circ} \mathrm{C}$ for $30 \mathrm{~s}$, $72{ }^{\circ} \mathrm{C}$ for $45 \mathrm{~s}$, and a final extension at $72^{\circ} \mathrm{C}$ for $10 \mathrm{~min}$. PCR product were extracted from a $2 \%$ agarose gel and further purified using the AxyPrep DNA Gel Extraction Kit (Axygen Biosciences, Union City, CA, USA) and quantified using QuantiFluor ${ }^{\mathrm{TM}}-\mathrm{ST}$ (Promega, USA) according to the manufacturer's protocol.

Purified amplicons were pooled in equimolar and paired-end sequenced $(2 \times 300)$ on an Illumina MiSeq platform (Illumina, San Diego, USA) according to the standard protocols by Majorbio Bio-Pharm Technology Co. Ltd. (Shanghai, China).

\section{Processing of sequencing data}

Raw fastq files were quality-filtered by Trimmomatic and merged by FLASH with the following criteria: (i) The reads were truncated at any site receiving an average quality core $<20$ over a 50 bp sliding window. (ii) Sequences whose overlap being longer than $10 \mathrm{bp}$ were merged according to their overlap with mismatch no more than 2 bp. (iii) Sequences of each sample were separated according to barcodes (exactly matching) and Primers (allowing 2 nucleotide mismatching), and reads containing ambiguous bases were removed.

Operational taxonomic units (OTUs) were clustered with $97 \%$ similarity cutoff using UPARSE (version 7.1 http://drive5.com/uparse/) with a novel 'greedy' algorithm that performs chimera filtering and OTU clustering simultaneously. The taxonomy of each $16 \mathrm{~S}$ rRNA gene sequence was analyzed by RDP Classifier algorithm (http://rdp.cme.msu.edu/) against the Silva (SSU123) $16 \mathrm{~S}$ rRNA database using confidence threshold of $70 \%$.

\section{Bioinformatics analysis}

QIIME Pipeline Version 1.9.1 [59] was used to analyze raw data. All reads were trimmed and then assigned to each sample based on their unique barcodes. After removing chimeras, all the reads were clustered into operational taxonomic units (OTUs) at a 97\% sequence identity, and were identified at different level of classification $[60,61]$. To standardize sampling efforts across samples, each sample was rarefied to the same number of reads $(22,494$ sequences). The rarefaction curves were generated based on the observed OTUs [62]. In addition, we calculated the shared and unique OTUs, phyla or genera between two zokor species. Shannon [63, 64], Chao [65], ACE [66] and Simpson [67] indices of gut microbiota were calculated by QIIME to evaluate the alpha diversity. We also investigated whether trapping location, sex, and body measurements affected the alpha diversity values. The body measurements including weight, body length, weight/body length and tail length were recorded for each sample. And we searched for correlations (Pearson with two-tailed significance tests) among the alpha diversity and body measurements. To assess beta diversity, principal coordinate analysis (PCoA) was performed based on Bray-Curtis and unweighted UniFrac distance to visualize the separation of gut microbiota structure across different species $[68,69]$.

\section{Statistical analysis}

Statistical analyses were conducted through SPSS 23.0 software $[70,71]$. The significant and the highly significant levels were 0.05 and 0.01 , respectively. The differences between species were detected by Wlicoxon rank sum test [72].

\section{Predicted metagenomes}

PICRUSTv1.1.0 [73] was used to predict the function based on the abundance of COGs. By comparing the 16S rRNA gene sequences with the reference genome database of microorganisms with known functions, the function can be predicted. Two-tailed t tests (Bonferroni corrected) were performed to test the differences of gene functions between $E$. cansus and E. rothschildi.

\section{Nucleotide sequence accession numbers}

The raw data of $16 \mathrm{~S}$ rRNA sequence were deposited into the NCBI Sequence Read Archive (SRA) database by accession number PRJNA664217 (http://www.ncbi.nlm. nih.gov/bioproject/664217).

\section{Supplementary Information}

The online version contains supplementary material available at https://doi. org/10.1186/s12917-021-02911-z.

Additional file 1 Table S1. The values of body measurements for each zokor sample. Table S2a. Correlation coefficients (and $p$ value) between 
alpha diversity values and a range of measures of $\mathrm{E}$. cansus. Significant effects are shown as ${ }^{* *} p<0.01,{ }^{*} p<0.05$. Table $\mathbf{S 2} \mathbf{b}$. Correlation coefficients (and $p$ value) between alpha diversity values and a range of measures of $E$. rothschildi. Significant effects are shown as ${ }^{* *} p<0.01$, * $p<0.05$. Table S3. The function of microbes of zokor based on COGs.

Additional file 2 Fig. $\mathbf{S 1}$ Rarefaction curves at OTU level (EC, E. cansus; $E R, E$. rothschildi). Fig. S2 Venn diagram showing the overlap of intestinal microbes between two zokor species (EC, E. cansus; ER, E. rothschildi). a. at OTU level. b. at phylum level. c. at genus level. Fig. S3 The comparisons of alpha diversity of intestinal microbes between two zokor species (EC, E. cansus; ER, E. rothschildi). a. Shannon diversity. b. Chao index. c. Ace index. d. Simpson diversity. Significant difference is indicated by asterisk. ${ }^{*}, p<0.05 ;{ }^{* *}, p<0.01 ;{ }^{* * *}, p<0.001$. Fig. S4 The comparisons of alpha diversity of intestinal microbes between males and females of E. cansus. Significant difference is indicated by asterisk. Fig. S5 The comparisons of alpha diversity of intestinal microbes between males and females of $E$. rothschildi. Significant difference is indicated by asterisk.

\section{Acknowledgements}

We sincerely thank Forest Disease and Pest Control Quarantine Station of Ningxia Hui Autonomous Region and Forest Pest Control Station of Hanzhong city for collecting samples of Eospalax cansus and Eospalax rothschildi, respectively.

\section{Authors' contributions}

All authors contributed to the study design. Sample collection and data analysis were performed by $Y Z, C X H$ and $X N N$. The first draft of the manuscript was written by $Y Z$. Experiment was performed by $Y Z$, NNL and XXZ. The revision suggestion of this paper was proposed by CXH and XNN All authors have read and approved the final manuscript.

\section{Funding}

This work was supported by the National Key Program of Research and Development (2017YFD0600103-4-1) and National Promoted Program of scientific and technological achievements in Forestry and Grassland (201929), and the Key Laboratory of Forestry and Grassland Administration in China on Management of Western Forest Bio-Disaster, Northwest Agriculture and Forestry University, Yangling 712100. The funding bodies provided all the help in the design of the study, sample collection, and analysis and interpretation of data.

\section{Availability of data and materials}

The original sequence data during the current study are available at the SRA by accession number PRJNA664217 (http://www.ncbi.nlm.nih.gov/ bioproject/664217).

\section{Declarations}

\section{Ethics approval and consent to participate}

This study was performed by strictly following Animal management regulations of the People's Republic of China. Processing of wild animals and sample collection were permitted by the local forestry departments. All experiments were approved and supervised by the Research Ethics Committee of Northwest Agriculture and Forestry University.

\section{Consent for publication}

Not applicable.

\section{Competing interests}

The authors declare that they have no competing interests.

Received: 14 January 2021 Accepted: 20 May 2021 Published online: 11 June 2021

\section{References}

1. Fan NC, Shi YZ. A revision of the zokors of subgenus Eospalax. Acta Theriologica Sinica. 1982;2:183-99.

2. Zhang Y, Zhang Z, Liu J. Burrowing rodents as ecosystem engineers: the ecology and management of plateau zokors in alpine meadow ecosystems on the Tibetan plateau. Mammal Rev. 2003;33(3-4):284-94. https://doi.org/1 0.1046/j.1365-2907.2003.00020.x.

3. Allen GM. The Mammals of China and Mongolia. Natural History of Central Asia, vol. 11. New York: The American Museum of Natural History; 1940. p. $1-620$.

4. Norris RW, Zhou KY, Zhou CQ, Yang G, William KC, Honeycutt RL. The phylogenetic position of the zokors (Myospalacinae) and comments on the families of muroids (Rodentia). Mol Phylogenet Evol. 2004;31(3):972-8. https://doi.org/10.1016/j.ympev.2003.10.020.

5. Flynn $\sqcup$. Chapter 4. The antiquity of Rhizomys and independent Acquisition of Fossorial Traits in subterranean Muroids. B Am Mus Nat Hist. 2009;331: 128-56. https://doi.org/10.1206/582-4.1.

6. Su J, Ji W, Wang J, Gleeson DM, Zhou JW, Hua LM, et al. Phylogenetic relationships of extant zokors (Myospalacinae) (Rodentia, Spalacidae) inferred from mitochondrial DNA sequences. Mitochondrial DNA. 2013;25(2):135-41.

7. Wang QY, Zhou WY, Wei WH, Zhang Y, Fan NC. The burrowing behavior of Myospalax baileyi and its relation to soil hardness. Acta Theriologica Sinica. 2000;20(4):277-83.

8. Lin GH. Subterranean rodents_- - the morphological adaptation of digging organs and the population genetics of Gansu zokor. Northwest Institute of Plateau Biology, Chinese Academy of Sciences, Doctoral dissertation, 2010.

9. Wang DH, Wang ZW. Seasonal variations in gastrointestinal tract morphology in plateau zokor (Myospalax baileyi). Acta Theriologica Sinica. 2000;20:270-6.

10. Wang Q, Yang ZJ, Li JG, He JP. Seasonal variations of morphological features and tissue structures of the digestive tract in Gansu zokor. Chinese Journal Of Zoology. 2016;51(4):573-82.

11. Maukonen J, Saarela M. Human gut microbiota: does diet matter? Proc Nutr Soc. 2015;74(1):23-36. https://doi.org/10.1017/S0029665114000688.

12. Murugesan S, Nirmalkar K, Hoyo-Vadillo C, Garcia-Espitia M, Ramirez-Sanchez D, Garcia-Mena J. Gut microbiome production of short-chain fatty acids and obesity in children. Eur J Clin Microbiol Infect Dis. 2017;37:621-5.

13. Shortt C, Hasselwander O, Meynier A, Nauta A, Fernandez EN, Putz P, et al. Systematic review of the effects of the intestinal microbiota on selected nutrients and non-nutrients. Eur J Nutr. 2017;57:25-49.

14. Sender R, Fuchs $S$, Milo R. Revised estimates for the number of human and bacteria cells in the body. PLoS Biol. 2016;14(8):e1002533. https://doi.org/1 0.1371/journal.pbio.1002533.

15. Nicholson JK, Holmes E, Kinross J, Bercelin R, Gibson G, Jia W, et al. Host-gut microbiota metabolic interactions. Science. 2012;336(6086):1262-7. https:// doi.org/10.1126/science.1223813.

16. Yang J, Nan XN, Zou Y, Zhang FR, Shi JN, Han CX. Effects of different factors on intestinal bacterial diversity of Eospalax cansus in the region of Liupan Mountains. Acta Microbiol Sin. 2018;58(08):1382-96.

17. Ren SE, Nan XN, Xu M, Zou Y, Liang NN, Shi JN, et al. Comparison of intestinal bacterial diversity of Gansu zokor under wild and artificial feeding conditions. Acta Microbiol Sin. 2020;60(04):826-38.

18. Zheng SH. Classification and evolution of the Siphneidae\|. In: Tomida Y, Li CK, Setoguchi T, editors. Rodent and Lagomorph Families of Asian Origins and Diversification. Tokyo: National Science Museum Monographs; 1994. p. 57-76.

19. Zou Y, Xu M, Ren SE, Liang NN, Han CX, Nan XN, et al. Taxonomy and phylogenetic relationship of zokors. J Genet. 2020;99(1). https://doi.org/10.1 007/s12041-020-01200-2.

20. Li BG, Chen FG. The classification of Eospalax. J Norwest Univ (nature science edition). 1992;22(3):339-44.

21. Li BG, Chen FG. The phylogeny, speciation and original center of Eospalax. J Norwest Univ (nature science edition). 1986;18(3):61-8.

22. Ley RE, Peterson DA, Gordon Jl. Ecological and evolutionary forces shaping microbial diversity in the human intestine. Cell. 2006;124(4):837-48. https:// doi.org/10.1016/j.cell.2006.02.017.

23. Steelman SM, Chowdhary BP, Dowd S, Suchodolski J, Janečka JE. Pyrosequencing of $16 \mathrm{~S}$ rRNA genes in fecal samples reveals high diversity of hindgut microflora in horses and potential links to chronic laminitis. BMC Vet Res. 2012;8(1):231. https://doi.org/10.1186/1746-6148-8-231.

24. Liu XF, Fan HL, Ding XB, Hong ZS, Nei YW, Liu ZW, et al. Analysis of the gut microbiota by high-throughput sequencing of the $\mathrm{V} 5-\mathrm{V} 6$ regions of the $16 \mathrm{~S}$ rRNA gene in donkey. Curr Microbiol. 2014;68(5):657-62. https://doi.org/10.1 007/s00284-014-0528-5.

25. Lagkouvardos I, Lesker TR, Hitch TCA, Galvez EJC, Smit N, Neuhaus K, et al. Sequence and cultivation study of Muribaculaceae reveals novel species, 
host preference, and functional potential of this yet undescribed family. Microbiome. 2019;7(1). https://doi.org/10.1186/s40168-019-0637-2.

26. Clarke SF, Murphy EF, O'Sullivan O, Lucey AJ, Humphreys M, Hogan A, et al. Exercise and associated dietary extremes impact on gut microbial diversity. Gut. 2014;63(12):1913-20. https://doi.org/10.1136/gutjnl-2013-306541.

27. Fndriks $L$. Roles of the gut in the metabolic syndrome: an overview. J Intern Med. 2017;281(4):319-36. https://doi.org/10.1111/joim.12584.

28. Werner JJ, Knights D, Garcia ML, Scalfonea NB, Smith S, Yarasheski K, et al. Bacterial community structures are unique and resilient in full-scale bioenergy systems. Proc Natl Acad Sci U S A. 2011;108(10):4158-63. https:// doi.org/10.1073/pnas.1015676108

29. Li H, Qu JP, Li TT, Wirth S, Zhang YM, Zhao XQ, et al. Diet simplification selects for high gut microbial diversity and strong fermenting ability in high-altitude pikas. Appl Microbiol Biotechnol. 2018;102(15):6739-51. https:// doi.org/10.1007/s00253-018-9097-z.

30. Zhang ZG, Xu DM, Wang L, Hao JJ, Wang JF, Zhou X, et al. Convergent evolution of rumen microbiomes in high-altitude mammals. Curr Biol. 2016; 26(14):1873-9. https://doi.org/10.1016/j.cub.2016.05.012

31. Weldon L, Abolins S, Lenzi L, Bourne C, Riley EM, Viney M. The gut microbiota of wild mice. PLoS One. 2015;10(8):e0134643. https://doi.org/1 0.1371/journal.pone.0134643.

32. Filippo CD, Cavalieri D, Paola MD, Ramazzotti M, Poullet JB, Massart S, et al. Impact of diet in shaping gut microbiota revealed by a comparative study in children from Europe and rural Africa. Proc Natl Acad Sci U S A. 2010; 107(33):14691-6. https://doi.org/10.1073/pnas.1005963107.

33. Fukuda S, Toh H, Hase K, Oshima K, Nakanishi Y, Yoshimura K, et al. Bifidobacteria can protect from enteropathogenic infection through production of acetate. Nature. 2011;469(7331):543-7. https://doi.org/10.103 8/nature09646.

34. Li ST, Zhang C, Gu YY, Chen L, Ou SY, Wang Y, et al. Lean rats gained more body weight than obese ones from a high-fibre diet. Br J Nutr. 2015;114(8): 1188-94. https://doi.org/10.1017/S0007114515002858.

35. Derrien M. Akkermansia muciniphila gen. Nov. sp. nov. a human intestinal mucin-degrading bacterium. Int J Syst Evol Microbiol. 2004;54(5):1469-76. https://doi.org/10.1099/ijs.0.02873-0.

36. Sekelja M, Berget I, Naes T, Rudi K. Unveiling an abundant core microbiota in the human adult colon by a phylogroup-independent searching approach. ISME J. 2011;5(3):519-31. https://doi.org/10.1038/ismej.2010.129.

37. Meehan CJ, Beiko RG. A Phylogenomic view of ecological specialization in the Lachnospiraceae, a family of digestive tract-associated Bacteria [J]. Genome Biol Evol. 2014;6(3):703-13. https://doi.org/10.1093/gbe/evu050.

38. Brulc JM, Antonopoulos DA, Miller ME, Wilson MK, Yannarell AC, Dinsdale EA, et al. Gene-centric metagenomics of the fiber-adherent bovine rumen microbiome reveals forage specific glycoside hydrolases. Proc Natl Acad Sci U S A. 2009;106(6):1948-53. https://doi.org/10.1073/pnas.0806191105.

39. Liu P, Zhao JB, Guo PT, Lu WQ, Geng ZY, Levesque CL, et al. Dietary corn bran fermented by Bacillus subtilis MA139 decreased gut cellulolytic Bacteria and microbiota diversity in finishing pigs. Front Cell Infect Microbiol. 2017;7: 526. https://doi.org/10.3389/fcimb.2017.00526.

40. Arntzen M, Anikó V, Mackie RI, Eijsink VGH, Pope PB. Outer membrane vesicles from Fibrobacter succinogenes $\mathrm{S} 85$ contain an array of carbohydrate-active enzymes with versatile polysaccharide-degrading capacity. Environ Microbiol. 2017;19(7):2701-14. https://doi.org/10.1111/1462-2920.13770.

41. Bensoussan L, Sarah M, Dassa B, Friedman N, Henrissat B, Lombard V, et al. Broad phylogeny and functionality of cellulosomal components in the bovine rumen microbiome. Environ Microbiol. 2016;19(1):185-97. https://doi. org/10.1111/1462-2920.13561.

42. Israeli-Ruimy V, Bule P, Jindou S, Dassa B, Morais S, Borovok L, et al. Complexity of the Ruminococcus flavefaciens FD-1 cellulosome reflects an expansion of family-related protein-protein interactions. Sci Rep. 2017;7(1): 42355. https://doi.org/10.1038/srep42355.

43. Moore WEC, Johnson JL, Holdeman LV. Emendation of Bacteroidaceae and Butyrivibrio and descriptions of Desulfomonas gen. Nov. and ten new species in the genera Desulfomonas, Butyrivibrio, Eubacterium, Clostridium, and Ruminococcus. Int J Syst Bacteriol. 1976;26(2):238-52. https://doi.org/1 0.1099/00207713-26-2-238.

44. Minj J, Chandra P, Paul C, Shama RK. Bio-functional properties of probiotic Lactobacillus: current applications and research perspectives. Crit Rev Food Sci Nutr. 2020:4:1-18.

45. Zhao JB, Liu P, Huang CF, Liu L, Li EK, Zhang G, et al. Effect of wheat bran on apparent total tract digestibility, growth performance, fecal microbiota and their metabolites in growing pigs. Anim Feed Sci Technol. 2018;239:1426. https://doi.org/10.1016/j.anifeedsci.2018.02.013.

46. Roager HM, Hansen LBS, Bahl MI, Frandsen HL, Carvalho V, Gøbel RJ, et al. Colonic transit time is related to bacterial metabolism and mucosal turnover in the gut. Nat Microbiol. 2016;1(9):16093. https://doi.org/10.1038/ nmicrobiol.2016.93.

47. Beaumont M, Portune KJ, Steuer N, Lan A, Cerrudo V, Audebert M, et al. Quantity and source of dietary protein influence metabolite production by gut microbiota and rectal mucosa gene expression: a randomized, parallel, double-blind trial in overweight humans. Am J Clin Nutr. 2017;106(4):100519. https://doi.org/10.3945/ajen.117.158816.

48. Manor O, Zubair N, Conomos MP, Xu X, Rohwer JE, Krafft CE, et al. A multiomic association study of trimethylamine N-oxide. Cell Rep. 2018;24(4): 935-46. https://doi.org/10.1016/j.celrep.2018.06.096.

49. Azcarate-Peril MA, Ritter AJ, Savaiano D, Monteagudo-Mera A, Anderson C, Magness ST, et al. Impact of short-chain galactooligosaccharides on the gut microbiome of lactose-intolerant individuals. Proc Natl Acad Sci U S A. 2017; 114(3):367-75.

50. Liu ZY. Isolation and identification of major cellulolytic bacteria in rumen of sheep and effects of nitrogen sources on their cellulolytic activities. Inner Mongolia agricultural university, Doctoral dissertation, 2008.

51. Scanlan PD, Shanahan F, Marchesi JR. Culture-independent analysis of desulfovibrios in the human distal colon of healthy, colorectal cancer and polypectomized individuals. FEMS Microbiol Ecol. 2009;69(2):213-21. https:// doi.org/10.1111/j.1574-6941.2009.00709.x.

52. Sasi Jyothsna TS, Tushar L, Sasikala C, Ramana CV. Paraclostridium benzoelyticum gen. Nov., sp. nov., isolated from marine sediment and reclassification of Clostridium bifermentans as Paraclostridium bifermentans comb. nov. proposal of a new genus Paeniclostridium gen. Nov. to accommodate Clostridium sordellii and Clostridium ghonii. Int J Syst Evol Microbiol. 2016;66(3):1268-74. https://doi.org/10.1099/ijsem.0.000874.

53. Sugie K, Komatsu T, Watando E, Inaba N, Kato K, Takamatsu D, et al. Septicemic Actinobacillus suis infection in a neonatal piglet with multifocal necrotic glossitis. J Vet Med Sci. 2019;81(2):274-8. https://doi.org/10.1292/ jvms.18-0630.

54. Teixeira LM, Merquior VLC, Shewmaker PL. Vagococcus. Encyclopedia of Food Microbiology (Second Edition). Academic Press; 2014. p. 673-9.

55. Gomes BP, Lilley JD, Drucker DB. Associations of endodontic symptoms and signs with particular combinations of specific bacteria. Int Endod J. 1996; 29(2):69-75. https://doi.org/10.1111/j.1365-2591.1996.tb01164.x.

56. Mamuad LL, Seo BJ, Faruk MSA, Espiritu HM, Jin SJ, Kim W, et al. Treponema spp the dominant pathogen in the lesion of bovine digital dermatitis and its characterization in dairy cattle. Vet Microbiol. 2020;245:108696.

57. Li H, Li TT, Yao MJ, Li JB, Zhang SH, Wirth S, et al. Pika gut may select for rare but diverse environmental Bacteria. Front Microbiol. 2016;7:1269.

58. Huang Z, Wang DD, Li JP, Wei C, He H. Transovarial Transmission of BacteriomeAssociated Symbionts in the Cicada Pycna repanda (Hemiptera: Cicadidae). Appl Environ Microbiol. 2020;86(12). https://doi.org/10.1128/AEM.02957-19.

59. Bokulich NA, Subramanian S, Faith JJ, Gevers D, Gordon JI, Knight R, et al. Quality-filtering vastly improves diversity estimates from Illumina amplicon sequencing. Nat Methods. 2013;10(1):11-57.

60. Blaxter M, Mann J, Chapman T, Thomas F, Whitton C, Floyd R, et al. Defining operational taxonomic units using DNA barcode data. Philos Trans R Soc B Biol Sci. 2005;360(1462):1935-43. https://doi.org/10.1098/rstb.2005.1725.

61. Li W, Godzik A. Cd-hit: a fast program for clustering and comparing large sets of protein or nucleotide sequences. Bioinformatics. 2006;22(13):1658-9. https://doi.org/10.1093/bioinformatics/btl158.

62. Kemp PF, Aller JY. Bacterial diversity in aquatic and other environments: what 165 rDNA libraries can tell us. FEMS Microbiol Ecol. 2004;47(2):161-77. https://doi.org/10.1016/S0168-6496(03)00257-5.

63. Shannon CE. A mathematical theory of communication. Bell Syst Tech J. 1948a;27(3):379-423. https://doi.org/10.1002/j.1538-7305.1948.tb01338.x.

64. Shannon CE. A mathematical theory of communication. Bell Syst Tech J. 1948b;27(4):623-56. https://doi.org/10.1002/j.1538-7305.1948.tb00917.x.

65. Chao A. Nonparametric estimation of the number of classes in a population. Scand J Stat. 1984;11:265-70.

66. Chao A, Yang MCK. Stopping rules and estimation for recapture debugging with unequal failure rates. Biometrika. 1993;80(1):193-201. https://doi.org/1 0.1093/biomet/80.1.193.

67. Simpson EH. Measurement of diversity. Nature. 1949;163(4148):688. https:// doi.org/10.1038/163688a0 
68. Ramette A. Multivariate analyses in microbial ecology. FEMS Microbiol Ecol. 2007;62(2):142-60. https://doi.org/10.1111/j.1574-6941.2007.00375.x.

69. Hamady M, Lozupone C, Knight R. Fast UniFrac: facilitating high-throughput phylogenetic analyses of microbial communities including analysis of pyrosequencing and PhyloChip data. ISME J. 2010;4(1):17-27. https://doi. org/10.1038/ismej.2009.97.

70. Xue W. Statistics analysis and the application of SPSS. Beijing: Chinese Renmin University Press; 2008. p. 1-136.

71. Feng YS. The application of SPSS 22.0. Beijing: Tsinghua University Press; 2015. p. 1-449.

72. Wilcoxon F. Individual comparisons by ranking methods. Biom Bull. 1945; 1(6):80-3. https://doi.org/10.2307/3001968.

73. Langille MG, Zaneveld J, Caporaso JG, McDonald D, Knights D, Reyes JA, et al. Predictive functional profiling of microbial communities using 165 rRNA marker gene sequences. Nat Biotechnol. 2013;31(9):814-21. https://doi. org/10.1038/nbt.2676.

\section{Publisher's Note}

Springer Nature remains neutral with regard to jurisdictional claims in published maps and institutional affiliations.

Ready to submit your research? Choose BMC and benefit from:

- fast, convenient online submission

- thorough peer review by experienced researchers in your field

- rapid publication on acceptance

- support for research data, including large and complex data types

- gold Open Access which fosters wider collaboration and increased citations

- maximum visibility for your research: over $100 \mathrm{M}$ website views per year

At $\mathrm{BMC}$, research is always in progress.

Learn more biomedcentral.com/submissions 